\title{
NORM INEQUALITIES FOR THE MINIMAL AND MAXIMAL OPERATOR, AND DIFFERENTIATION OF THE INTEGRAL
}

\author{
David Cruz-Uribe, SFO, C. J. Neugebauer and V. Olesen
}

\begin{abstract}
We study the weighted norm inequalities for the minimal operator, a new operator analogous to the Hardy-Littlewood maximal operator which arose in the study of reverse Hölder inequalities. We characterize the classes of weights which govern the strong and weak-type norm inequalities for the minimal operator in the two weight case, and show that these classes are the same. We also show that a generalization of the minimal operator can be used to obtain information about the differentiability of the integral in cases when the associated maximal operator is large, and we give a new condition for this maximal operator to be weak $(1,1)$.
\end{abstract}

\section{Introduction}

Given a real-valued, measurable function $f$ on $\mathbb{R}^{n}$, the HardyLittlewood maximal function of $f$ is defined by

$$
M f(x)=\sup \frac{1}{|I|} \int_{I}|f| d y,
$$

where the supremum is taken over all cubes $I$ which contain $x$ and whose sides are parallel to the co-ordinate axes. Intuitively, the maximal operator controls where the function $f$ is large. We define an analogous operator, the minimal operator, which controls where a function is small. Given a function $f$, define the minimal function of $f$ by

$$
m f(x)=\inf \frac{1}{|I|} \int_{I}|f| d y,
$$

Keywords. Minimal operator, maximal operator, weighted norm inequalities, differentiation of the integral.

1991 Mathematics subject classifications: 42B25. 
where again the infimum is taken over all cubes which contain $x$. By the Lebesgue differentiation theorem, if $f$ is locally integrable then $m f(x) \leq|f(x)| \leq M f(x)$ a.e.

The first two authors introduced the minimal operator in [2], where we used it to study the fine structure of functions which satisfy the reverse Hölder inequality. We also examined the one-weight, weighted norm inequalities which it satisfies. Further, we have used the minimal operator to study one-sided reverse Hölder inequalities and one-sided $\left(A_{p}\right)$ weights [4], and to study the convergence of convolution operators with "bad" kernels [5]. In a forthcoming paper [3] we apply the weighted norm inequalities for the minimal operator (including the results in this paper) to study the weighted norm inequalities for the geometric maximal operator

$$
M_{0} f(x)=\sup _{I} \exp \left(\frac{1}{|I|} \int_{I} \log |f(y)| d y\right),
$$

which have been studied previously by Sbordone and Wik $[\mathbf{2 0}]$ and Yin and Muckenhoupt [27].

The purpose of this paper is to study the two-weight, weighted norm inequalities for the minimal operator. As we showed in [2], this problem is complicated by the fact that, unlike the maximal operator, the natural domain of the minimal operator is not $L^{p}$ : if $f \in L^{p}, p<\infty$, then $m f \equiv 0$. Following up on the idea that the minimal operator controls where a function is small, we showed there that the minimal operator has two natural domains: functions $f$ such that either $\log |f|$ or $1 / f$ is in $L^{p}$. The latter case is much more interesting, and the main result in this paper is the following theorem.

Theorem 1.1. Given $p>0$ and a pair of non-negative weights $(u, v)$ on $\mathbb{R}$, the following are equivalent:

1. $(u, v) \in\left(W_{p}\right)$ : there exists a constant $C$ such that given any interval $I \subset \mathbb{R}$

$$
\frac{1}{|I|} \int_{I} u d x \leq C\left(\frac{1}{|I|} \int_{I} v^{1 /(p+1)} d x\right)^{p+1} ;
$$

2. the weak-type inequality

$$
u(\{x: m f(x)<1 / t\}) \leq \frac{C}{t^{p}} \int_{\mathbb{R}} \frac{v}{|f|^{p}} d x
$$

holds for every $f$ such that $1 / f$ is in $L^{p}(v)$; 
3. $(u, v) \in\left(W_{p}\right)^{*}$ : there exists a constant $C$ such that given any interval $I \subset \mathbb{R}$,

$$
\int_{I} \frac{u}{m\left(\sigma / \chi_{I}\right)^{p}} d x \leq C \int_{I} \sigma d x
$$

where $\sigma=v^{1 /(p+1)}$;

4. the strong-type inequality

$$
\int_{\mathbb{R}} \frac{u}{(m f)^{p}} d x \leq C \int_{\mathbb{R}} \frac{v}{|f|^{p}} d x
$$

holds for every $f$ such that $1 / f$ is in $L^{p}(v)$.

The equivalence of (1) and (2) and of (3) and (4) in Theorem 1.1 is very similar to results which hold for the maximal operator, with the Muckenhoupt class $\left(A_{p}\right)$ replaced by $\left(W_{p}\right)$ and the Sawyer class $\left(S_{p}\right)$ replaced by $\left(W_{p}\right)^{*}$. (Recall that $\left(S_{p}\right)$ is defined by

$$
\left.\int_{I} M\left(v^{1-p^{\prime}} \chi_{I}\right)^{p} u d x \leq C \int_{I} v^{1-p^{\prime}} d x<+\infty .\right)
$$

However, this theorem has two surprising features. First, the norm inequalities hold for all $p>0$, and not just for $p \geq 1$. For the maximal operator, it is well known that if $f \in L^{1}$ then $M f$ is never in $L^{1}$ unless $f \equiv 0$. (See, for example, Stein [23].) Second, the weight classes $\left(W_{p}\right)$ and $\left(W_{p}\right)^{*}$ are the same. For the maximal operator, $\left(A_{p}\right)$ is strictly larger than $\left(S_{p}\right)$. (For an elementary example, see García-Cuerva and Rubio de Francia [6].)

We have only been able to prove Theorem 1.1 in full generality on the real line. The central difficulty is that the minimal operator is not equivalent to the centered minimal operator, $m_{c}$, defined as the minimal operator except the infimum is taken over cubes whose center is $x$. (An easy example is given by $f(x)=e^{x}$. By contrast, the maximal operator and the centered maximal operator are equivalent.) Therefore, when proving weighted norm inequalities we are unable to use the Besicovitch covering lemma. On $\mathbb{R}$ there are special covering lemmas which let us overcome this problem. In higher dimensions we can prove some partial extensions, but only if we assume doubling conditions on the weights. This leads to two open questions.

Question 1.2. Does Theorem 1.1 hold in higher dimensions in full generality, or are doubling conditions necessary?

For the possible necessity of doubling conditions in higher dimensions, it is worthwhile to consider the behavior of the weighted maximal function on $\mathbb{R}$ and $\mathbb{R}^{n}, n>1$. (See Sjögren [22] and Vargas [26].) 
Question 1.3. What are the weights which govern the weighted norm inequalities for the centered minimal operator?

Following the proof of Theorem 1.1, it is easy to see that for $p>0$, a necessary condition on $(u, v)$ for the centered minimal operator to satisfy a weak-type inequality is a "weak" $\left(W_{p}\right)$ condition: for every cube $I$,

$$
\frac{1}{|I|} \int_{I} u d x \leq C\left(\frac{1}{|2 I|} \int_{2 I} v^{1 /(p+1)} d x\right)^{p+1},
$$

where $2 I$ is the cube with the same center as $I$ and twice the side-length. We conjecture that this condition is also sufficient. If true, this result would be especially interesting in the one-weight case, since in that case this condition is equivalent to a weak reverse Hölder inequality which appears frequently in the study of PDE's and quasi-conformal mappings. (See, for example, Sawyer [19] or Iwaniec and Nolder [10].)

The proof of Theorem 1.1 is organized as follows: in Section 2 we show that the $\left(W_{p}\right)$ condition is equivalent to the weak-type inequality; in Section 3 we show that the $\left(W_{p}\right)^{*}$ condition is equivalent to the strong-type inequality; and in Section 4 we show that the classes $\left(W_{p}\right)$ and $\left(W_{p}\right)^{*}$ are the same. At the end of each section we describe briefly how these results can be extended to higher dimensions. At the end of Section 3 we also show that, again unlike the maximal operator, mixed norm inequalities hold only trivially for the minimal operator. (See Sawyer [18].)

In Section 5 we examine the properties of the $\left(W_{p}\right)$ weights, especially their relation to the $\left(A_{p}\right)$ weights. We have the formal relationship that $\left(W_{p}\right)=\left(A_{-p}\right)$, that is, the $\left(A_{p}\right)$ condition with $p$ replaced by $-p, p>0$. We also have the chain of proper inclusions

$$
\left(A_{1}\right) \subset\left(A_{r}\right) \subset\left(A_{s}\right) \subset\left(W_{\infty}\right) \subset\left(W_{p}\right) \subset\left(W_{q}\right),
$$

for all $1<r<s, 0<q<p$. (The class $\left(W_{\infty}\right)$ will be defined below.) These results are mostly elementary - the full structure of $\left(W_{p}\right)$ weights remains to be determined.

Sections 6 and 7 contain applications of a generalization of the minimal operator to problems of differentiation of the integral. Let $\mathcal{B}=\left\{\mathcal{B}_{x}: x \in \mathbb{R}^{n}\right\}$ be a differentiation basis: for each $x, \mathcal{B}_{x}=\left\{E_{j x}\right\}$, where the sets $E_{j x} \rightarrow x$-that is, for every $\epsilon>0, E_{j x} \subset\{t:|t-x| \leq \epsilon\}$ for all $j$ sufficiently large. Note that this is more general than the usual definition since we do not assume that $x \in E_{j x}$ or that $E_{j x}$ is open. Define the maximal operator relative to $\mathcal{B}$ by

$$
M_{\mathcal{B}} f(x)=\sup _{\mathcal{B}_{x}} \frac{1}{\left|E_{j x}\right|} \int_{E_{j x}}|f| d y .
$$


We will assume that $\mathcal{B}$ is such that $M_{\mathcal{B}}$ is measurable; this property is easy to check for the classical differentiation bases. (See de Guzmán [7].) If $M_{\mathcal{B}}$ is weak-type $(p, p)$ for some $1 \leq p<\infty$, then it is known that $\mathcal{B}$ differentiates $L^{p}$ : given a function $f$ in $L^{p}$, the exceptional set

$$
E_{\mathcal{B}}(f)=\left\{x: \limsup _{j \rightarrow \infty}\left|\frac{1}{\left|E_{j x}\right|} \int_{E_{j x}} f d y-f(x)\right|>0\right\}
$$

has measure zero. (See de Guzmán [7], [8].) In Section 6 we consider the case when $M_{\mathcal{B}}$ is weak-type $(p, p)$ but $f$ is not in $L^{p}$. Using the minimal operator, we develop a type of convergence such that if a sequence $\left\{g_{k}\right\}$ converges to $f$ then the measure of the exceptional set $E_{\mathcal{B}}(f)$ is controlled by the measures of the $E_{\mathcal{B}}\left(g_{k}\right)$ 's. This can be thought of as a generalization of a result by Hayes and Pauc [9] on the "transmission" of differentiability. (Also see de Guzmán [7], [8].) Our approach appears to be different from earlier work in this area and needs to be explored further.

Because of the importance of the assumption that $M_{\mathcal{B}}$ be weak-type $(p, p)$ for some $p$, in Section 7 we give a new result on the weak-type behavior of $M_{\mathcal{B}}$ in the case where each $\mathcal{B}_{x}$ is the translate by $x$ of a fixed collection $\left\{E_{j}\right\}$. As a corollary we generalize results of Nagel and Stein [16], [24]: if the sets $E_{j}=\left\{x:\left|x-x_{j}\right| \leq r_{j}\right\}$ tend to zero (whether or not $\left.\left|x_{j}\right| / r_{j} \rightarrow \infty\right)$ then there is a subsequence $\mathcal{B}=\left\{E_{j_{k}}\right\}$ such that the maximal operator $M_{\mathcal{B}}$ is weak $(1,1)$.

Throughout this paper all notation is standard or will be defined as needed. All cubes are assumed to have their sides parallel to the coordinate axes. Given a Borel set $E$ and a function $f$, let $|E|$ denote the Lebesgue measure of $E, f(E)=\int_{E} f d x$ and $E(f)=f(E) /|E|$. By $f / \chi_{E}$ we denote the function equal to $f$ on $E$ and infinity elsewhere. The letter $C$ will denote a positive constant whose value may change at each appearance.

Finally, we want to thank the referee for the many insightful corrections and comments contained in the report.

\section{Weak-Type Norm Inequalities}

In this section we prove that statements (1) and (2) in Theorem 1.1 are equivalent. The proof requires two classical covering lemmas which are special to $\mathbb{R}$. We sketch their proofs as a convenience to the reader.

Lemma 2.1. Let $\mathcal{F}$ be a collection of intervals in $\mathbb{R}$. Then there exists a countable subcollection $\mathcal{F}_{0}$ such that $\bigcup\{I: I \in \mathcal{F}\}=\bigcup\left\{I: I \in \mathcal{F}_{0}\right\}$. 
Proof: If $I \in \mathcal{F}$, let $I^{o}$ denote the interior of $I$. Then we can find a countable subcollection $\mathcal{F}_{1}$ such that $\bigcup\left\{I^{o}: I \in \mathcal{F}\right\}=\bigcup\left\{I^{o}: I \in \mathcal{F}_{1}\right\}$. To finish the proof, note that the set $\bigcup\{I: I \in \mathcal{F}\} \backslash \bigcup\left\{I^{\circ}: I \in \mathcal{F}\right\}$ must be countable.

Lemma 2.2. Let $\mathcal{F}$ be a finite collection of intervals in $\mathbb{R}$. Then there exist two subcollections $\mathcal{F}_{1}$ and $\mathcal{F}_{2}$, each consisting of pairwise disjoint intervals, such that $\bigcup\{I: I \in \mathcal{F}\}=\bigcup\left\{I: I \in \mathcal{F}_{1} \cup \mathcal{F}_{2}\right\}$.

Proof: Let $\mathcal{F}=\left\{I_{j}, 1 \leq j \leq N\right\}$. Without loss of generality, we may assume that each $I_{j}$ contains a point $x_{j}$ not contained in any other element of $\mathcal{F}$. By re-ordering the elements of $\mathcal{F}$ we may also assume that $x_{1}<x_{2}<\cdots<x_{N}$. Now let $\mathcal{F}_{1}=\left\{I_{1}, I_{3}, \ldots\right\}, \mathcal{F}_{2}=\left\{I_{2}, I_{4}, \ldots\right\}$.

To prove that (1) implies (2), fix non-negative $f$ such that $1 / f \in L^{p}(v)$. We first assume that $f(x)$ is everywhere positive. For each $t>0$ let $E_{t}=\{x: m f(x)<1 / t\}$. Then for each $x \in E_{t}$ there exists an interval $I_{x}$ containing $x$ such that

$$
\frac{1}{\left|I_{x}\right|} \int_{I_{x}} f d y<1 / t .
$$

Clearly $I_{x} \subset E_{t}$, so $E_{t}$ is equal to the union of the $I_{x}$ 's. By Lemma 2.1 $\left\{I_{x}\right\}$ has a countable subcollection $\left\{I_{j}\right\}$ whose union is $E_{t}$. Let $E_{N}=\bigcup\left\{I_{j}: 1 \leq j \leq N\right\}$ and apply Lemma 2.2 to this finite collection. This gives us a disjoint subcollection $\mathcal{F}_{N}$ of $\left\{I_{j}: 1 \leq j \leq N\right\}$ such that

$$
u\left(E_{N}\right) \leq 2 \sum_{I \in \mathcal{F}_{N}} u(I)
$$

If we combine this with inequality $(1)$ and the $\left(W_{p}\right)$ condition we see that, since $f(I)>0$,

$$
\begin{aligned}
u\left(E_{N}\right) \leq \frac{2}{t^{p}} \sum_{I \in \mathcal{F}_{N}} u(I) & \left(\frac{1}{|I|} \int_{I} f d x\right)^{-p} \\
& \leq \frac{C}{t^{p}} \sum_{I \in \mathcal{F}_{N}}\left(\int_{I} v^{1 /(p+1)} d x\right)^{p+1}\left(\int_{I} f d x\right)^{-p} .
\end{aligned}
$$

By Hölder's inequality,

$$
\begin{aligned}
& \int_{I} v^{1 /(p+1)} d x=\int_{I} \frac{v^{1 /(p+1)}}{f^{p /(p+1)}} \cdot f^{p /(p+1)} d x \\
& \leq\left(\int_{I} \frac{v}{f^{p}} d x\right)^{1 /(p+1)}\left(\int_{I} f d x\right)^{p /(p+1)} .
\end{aligned}
$$


If we combine this with the fact that the intervals in $\mathcal{F}_{N}$ are disjoint, we see that

$$
u\left(E_{N}\right) \leq \frac{C}{t^{p}} \int_{\mathbb{R}} \frac{v}{f^{p}} d x .
$$

The constant $C$ is independent of $N$, so the weak-type inequality follows if we let $N$ tend to infinity.

Now for arbitrary $f$ define the sequence $f_{n}=f+1 / n$. Then each $f_{n}$ is strictly positive and $1 / f_{n} \in L^{p}(v)$ if $1 / f$ is. Further, a straightforward argument shows that the $m f_{n}(x)$ 's decrease to $m f(x)$. By the above argument the weak-type inequality holds for each $f_{n}$, so by the monotone convergence theorem it holds for arbitrary $f$.

To show that (2) implies (1) is straightforward. Fix an interval $I$ and let $f=v^{1 /(p+1)} / \chi_{I}$. If $v^{1 /(p+1)}(I)=+\infty$ then (1) is immediate. If it is finite, then for $x \in I$,

$$
m f(x) \leq \frac{1}{|I|} \int_{I} v^{1 /(p+1)} d x \equiv 1 / t .
$$

If we substitute this into the weak-type inequality we get

$$
u(I) \leq C\left(\frac{1}{|I|} \int_{I} v^{1 /(p+1)} d x\right)^{p} \cdot \int_{I} v^{1-p /(p+1)} d x,
$$

and this is the $\left(W_{p}\right)$ condition.

Remark. In higher dimensions, the $\left(W_{p}\right)$ condition is still necessary for the weights $(u, v)$ to satisfy the weak-type inequality; the above proof goes through without change. We can prove that it is sufficient if we assume that $u$ is a doubling weight: there exists a constant $C$ such that $u(2 I) \leq C u(I)$ for every cube $I$. To adapt the proof, note that $E_{t} \subset \bigcup\left\{2 I_{x}: x \in E_{t}\right\}$, and hence by the Besicovitch covering theorem $E_{t} \subset \cup 2 I_{j}$, where the $I_{j}$ 's have bounded overlap and $t \int_{I_{j}} f d x \leq\left|I_{j}\right|$. The rest of the proof goes through without change.

\section{Strong-Type Norm Inequalities}

In this section we prove that conditions (3) and (4) in Theorem 1.1 are equivalent. To do so, we need to introduce two auxiliary operators. Let $\sigma$ be a Borel measure on $\mathbb{R}$. Define the weighted maximal and minimal operators

$$
M_{\sigma} f(x)=\sup \frac{1}{\sigma(I)} \int_{I}|f| d \sigma, \quad m_{\sigma} f(x)=\inf \frac{1}{\sigma(I)} \int_{I}|f| d \sigma,
$$


where the supremum and infimum are taken over all $I$ containing $x$ such that $\sigma(I)>0$. For $1<p \leq \infty,\left\|M_{\sigma} f\right\|_{p, \sigma} \leq C_{p}\|f\|_{p, \sigma}$ (See Sjögren [22]. Also, this can be proved by using Lemma 2.2 to obtain a weak $(1,1)$ inequality and then using interpolation to get the strong-type inequality.) We will use this fact to prove a norm inequality for the weighted minimal operator.

Lemma 3.1. There exists a constant $C$ such that for every $0<p<\infty$ and $f$ such that $1 / f \in L^{p}(\sigma)$,

$$
\int_{\mathbb{R}} \frac{d \sigma}{\left(m_{\sigma} f\right)^{p}} \leq C \int_{\mathbb{R}} \frac{d \sigma}{|f|^{p}} .
$$

Proof: Fix $0<p<\infty$ and $r>1$ such that $(r-1) p=2$. By Hölder's inequality, $\left(m_{\sigma} f\right)^{-1} \leq M_{\sigma}\left(f^{1-r^{\prime}}\right)^{r-1}$. Then by the above remark,

$$
\begin{aligned}
\int \frac{d \sigma}{\left(m_{\sigma} f\right)^{p}} \leq \int M_{\sigma}\left(f^{1-r^{\prime}}\right)^{(r-1) p} d \sigma & \\
& \leq C \int\left(|f|^{1-r^{\prime}}\right)^{(r-1) p} d \sigma=C \int \frac{d \sigma}{|f|^{p}} .
\end{aligned}
$$

We also need to show that the $\left(W_{p}\right)^{*}$ condition implies an apparently stronger condition.

Lemma 3.2. If $(u, v) \in\left(W_{p}\right)^{*}$ then there exists a constant $C$ such that if $F$ is a finite union of intervals, then

$$
\int_{F} \frac{u}{m\left(\sigma / \chi_{F}\right)^{p}} d x \leq C \sigma(F)
$$

Proof: If $I$ and $J$ are disjoint, closed intervals, then for each $x \in I$, $m\left(\sigma / \chi_{I \cup J}\right)(x)=m\left(\sigma / \chi_{I}\right)(x)$. For if $K$ is an interval, $x \in K$ and $|K \backslash I|>0$, then

$$
\frac{1}{|K|} \int_{K} \frac{\sigma}{\chi_{I \cup J}}=\infty .
$$

Therefore, when calculating $m\left(\sigma / \chi_{I \cup J}\right)(x)$ for $x \in I$, we can restrict ourselves to intervals $K$ such that $K \subset I$. Hence

$$
\begin{aligned}
\int_{I \cup J} \frac{u}{m\left(\sigma / \chi_{I \cup J}\right)^{p}} d x & =\int_{I} \frac{u}{m\left(\sigma / \chi_{I}\right)^{p}} d x+\int_{J} \frac{u}{m\left(\sigma / \chi_{J}\right)^{p}} d x \\
& \leq C(\sigma(I)+\sigma(J)) \\
& =C \sigma(I \cup J) .
\end{aligned}
$$


In general, if $F$ is a finite union of intervals then $F$ is (up to a set of measure zero) the finite union of disjoint, closed intervals, and the proof follows by induction.

We can now prove the desired result. For any $p>0$, the proof that (4) implies (3) is essentially the same as the proof that (2) implies (1): let $f=v^{1 /(p+1)} / \chi_{I}$ and substitute this into the strong-type inequality. The $\left(W_{p}\right)^{*}$ condition follows at once.

The proof that (3) implies (4) is similar to the proof of the strongtype inequality for the maximal operator given by Jawerth [11]. We first assume that $v$ is everywhere positive; we treat the general case at the end. Fix $p>0$ and $f$ non-negative such that $1 / f \in L^{p}(v)$.

For each integer $k$, let $A_{k}=\left\{x: 2^{-(k+1)} \leq m f(x)<2^{-k}\right\}$ and let $K_{k}$ be a compact subset of $A_{k}$. We can cover $K_{k}$ by a finite collection of intervals $I_{j}^{k}, 1 \leq j \leq j_{k}$, such that

$$
\frac{1}{2^{k+1}} \leq \frac{1}{\left|I_{j}^{k}\right|} \int_{I_{j}^{k}} f d x<\frac{1}{2^{k}}
$$

By induction, define the disjoint sets $E_{1}^{k}=I_{1}^{k} \cap K_{k}, E_{2}^{k}=\left(I_{2}^{k} \backslash I_{1}^{k}\right) \cap$ $K_{k}, \ldots$. Then $K_{k}=\cup E_{j}^{k}$. Given an integer $N>0$, we have

$$
\begin{aligned}
\int_{\cup_{-N}^{N} K_{k}} \frac{u}{(m f)^{p}} d x & =\sum_{k, j} \int_{E_{j}^{k}} \frac{u}{(m f)^{p}} d x \\
& \leq 2^{p} \sum_{k, j} 2^{k p} u\left(E_{j}^{k}\right) \\
& \leq 2^{p} \sum_{k, j} u\left(E_{j}^{k}\right)\left|I_{j}^{k}\right|^{p}\left(\int_{I_{j}^{k}} f d x\right)^{-p} .
\end{aligned}
$$

Since $v$ is positive, $\sigma\left(I_{j}^{k}\right)>0$. Further, by Hölder's inequality,

$$
\begin{aligned}
\sigma\left(I_{j}^{k}\right) & \leq\left(\int_{\mathbb{R}} \frac{v}{f^{p}} d x\right)^{1 /(p+1)}\left(\int_{I_{j}^{k}} f d x\right)^{p /(p+1)} \\
& \leq\left(\int_{\mathbb{R}} \frac{v}{f^{p}} d x\right)^{1 /(p+1)}\left(2^{-k}\left|I_{j}^{k}\right|\right)^{p /(p+1)} \\
& <+\infty
\end{aligned}
$$


Therefore,

$$
\begin{aligned}
2^{p} \sum_{k, j} u\left(E_{j}^{k}\right)\left|I_{j}^{k}\right|^{p} & \left(\int_{I_{j}^{k}} f d x\right)^{-p} \\
& =2^{p} \sum_{k, j} u\left(E_{j}^{k}\right)\left(\frac{\left|I_{j}^{k}\right|}{\sigma\left(I_{j}^{k}\right)}\right)^{p}\left(\frac{1}{\sigma\left(I_{j}^{k}\right)} \int_{I_{j}^{k}} \frac{f}{\sigma} \cdot \sigma d x\right)^{-p} .
\end{aligned}
$$

Let $\mu$ be the measure on $\mathbb{Z} \times\{-N, \ldots, N\}$ defined by

$$
\mu(j, k)=\frac{u\left(E_{j}^{k}\right)\left|I_{j}^{k}\right|^{p}}{\sigma\left(I_{j}^{k}\right)^{p}}
$$

if $j \leq j_{k}$, and $\mu(j, k)=0$ if $j>j_{k}$. For $\lambda>0$ define

$$
\begin{aligned}
& \Gamma(\lambda)=\left\{(j, k):-N \leq k \leq N, 1 \leq j \leq j_{k}\right. \\
& \text { and } \left.\left(\frac{1}{\sigma\left(I_{j}^{k}\right)} \int_{I_{j}^{k}} \frac{f}{\sigma} \sigma d x\right)^{p}<1 / \lambda\right\},
\end{aligned}
$$

and let $G(\lambda)=\bigcup I_{j}^{k}$, where the union is taken over all $(j, k) \in \Gamma(\lambda)$. We can now rewrite the above inequality as

$$
\begin{aligned}
\int_{\cup_{-N}^{N} K_{k}} \frac{u}{(m f)^{p}} d x \leq 2^{p} \sum_{j, k} \mu(j, k)\left(\frac{1}{\sigma\left(I_{j}^{k}\right)} \int_{I_{j}^{k}} \frac{f}{\sigma} \sigma d x\right)^{-p} & \\
& =2^{p} \int_{0}^{\infty} \mu(\Gamma(\lambda)) d \lambda .
\end{aligned}
$$

Using Lemma 3.2 we estimate the integrand of the right-hand side as 
follows:

$$
\begin{aligned}
\mu(\Gamma(\lambda)) & =\sum_{\Gamma(\lambda)} \frac{u\left(E_{j}^{k}\right)\left|I_{j}^{k}\right|^{p}}{\sigma\left(I_{j}^{k}\right)^{p}} \\
& \leq \sum_{\Gamma(\lambda)} u\left(E_{j}^{k}\right) \inf _{x \in E_{j}^{k}}\left(m\left(\sigma / \chi_{I_{j}^{k}}\right)(x)\right)^{-p} \\
& \leq \sum_{\Gamma(\lambda)} \int_{E_{j}^{k}} \frac{u}{m\left(\sigma / \chi_{I_{j}^{k}}\right)^{p}} d x \\
& \leq \sum_{\Gamma(\lambda)} \int_{E_{j}^{k}} \frac{u}{m\left(\sigma / \chi_{G(\lambda)}\right)^{p}} d x \\
& \leq \int_{G(\lambda)} \frac{u}{m\left(\sigma / \chi_{G(\lambda)}\right)^{p}} d x \\
& \leq C \sigma(G(\lambda)) \\
& \leq C \sigma\left\{x: m_{\sigma}(f / \sigma)(x)^{p}<1 / \lambda\right\} .
\end{aligned}
$$

Therefore, by Lemma 3.1,

$$
\begin{aligned}
\int_{\cup_{-N}^{N} K_{k}} \frac{u}{(m f)^{p}} d x & \leq 2^{p} C \int_{0}^{\infty} \sigma\left\{m_{\sigma}(f / \sigma)^{p}<1 / \lambda\right\} d \lambda \\
& =2^{p} C \int_{\mathbb{R}} \frac{\sigma}{m_{\sigma}(f / \sigma)^{p}} d x \\
& \leq C \int_{\mathbb{R}} \frac{v}{f^{p}} d x
\end{aligned}
$$

To complete the proof, note that we can find a nested sequence of compact sets $K_{k, n}$ which increase to $A_{k}$. Since in the above argument the $K_{k}$ 's were arbitrary compact sets, by the monotone convergence theorem

$$
\int_{\cup_{-N}^{N} A_{k}} \frac{u}{(m f)^{p}} d x \leq C \int_{\mathbb{R}} \frac{v}{f^{p}} d x .
$$

If we let $N$ tend to $\infty$ then, again by the monotone convergence theorem, we get

$$
\int_{\cup A_{k}} \frac{u}{(m f)^{p}} d x \leq C \int_{\mathbb{R}} \frac{v}{f^{p}} d x .
$$


This gives us the weak-type inequality (for positive $v$ ) provided that

$$
u\left(\mathbb{R} \backslash \bigcup A_{k}\right)=u(\{x: m f(x)=0\})=0 .
$$

But since $(u, v) \in\left(W_{p}\right)^{*},(u, v) \in\left(W_{p}\right)$ (see Section 4 below), so this follows from inequality (2) in Theorem 1.1.

Finally, for general $v$ note that if $(u, v) \in\left(W_{p}\right)^{*}$ then $(u, v+\epsilon) \in\left(W_{p}\right)^{*}$ with constant independent of $\epsilon$. By the above argument the strong-type inequality holds for $(u, v+\epsilon)$ and it follows for $(u, v)$ by letting $\epsilon$ tend to 0 .

Remarks. In the proof of the strong-type inequality the constant $C$ is at most the product of the $\left(W_{p}\right)^{*}$ constant of $(u, v)$, the constant of Lemma 3.1 and $2^{p}$. However, if in the definition of the sets $A_{k}$ we replace $2^{k}$ by $a^{k}, a>1$, then the argument goes through as before, only now the constant contains a factor $a^{p}$. Taking the limit as $a$ tends to 1 we see that the constant in the strong-type norm inequality depends only on the $\left(W_{p}\right)^{*}$ constant and the constant from Lemma 3.1.

The equivalence of conditions (3) and (4) in Theorem 1.1 extends to higher dimensions but not in a satisfactory manner. First note that the proof that (4) implies (3) goes through without change, so the $\left(W_{p}\right)^{*}$ condition is still necessary.

Second, if we assume that $\sigma$ is doubling, then in $\mathbb{R}^{n} M_{\sigma}$ is a bounded operator from $L^{p}(\sigma)$ into itself, $p>1$. (See, for example García-Cuerva $[6]$.) Therefore Lemma 3.1 still holds. Note, however, that this is a different doubling condition than the one necessary to extend the proof of the weak-type inequality to higher dimensions. Further, we need the doubling condition on $\sigma$, and not on $v$ itself. As we will show below (see Section 5) if $(u, v) \in\left(W_{p}\right)^{*}$ then $(u, v) \in\left(W_{q}\right)^{*}$ for all $q<p$. However, it is not known whether $v^{1 /(p+1)}$ being a doubling weight implies that $v^{1 /(q+1)}$ is one as well. This leads to the following open question, which we believe is interesting in its own right.

Question 3.3. If $w$ is a doubling weight on $\mathbb{R}^{n}$, is $w^{r}$ for all $0<r<1$ ?

This is true if $w \in\left(A_{\infty}\right)$, but it is unclear if it is true in general.

Finally, the proof of Lemma 3.2 depends even more heavily on the geometry of $\mathbb{R}$, and does not extend to higher dimensions. Therefore, in $\mathbb{R}^{n}$ we need to replace the $\left(W_{p}\right)^{*}$ condition with the stronger condition of Lemma 3.2. 
We end this section with the following observation: unlike the maximal operator, mixed norm inequalities hold for the minimal operator only trivially.

Theorem 3.4. Let $0<p<q<\infty$ and suppose that for all nonnegative $f$

$$
u\{x: m f(x)<1 / t\} \leq \frac{C}{t^{q}}\left(\int_{\mathbb{R}} \frac{v}{f^{p}} d x\right)^{q / p} .
$$

Then either the left-hand side equals zero or the right-hand side equals infinity.

Proof: We first show that if for some interval $I_{0}, \int_{I_{0}} v^{1 /(p+1)} d x<$ $\infty$, then $u(x)=0$ for almost every $x \in I_{0}$. Let $I \subset I_{0}$ and let $f=v^{1 /(p+1)} / \chi_{I}$. Then for $x \in I$,

$$
m f(x) \leq \frac{1}{|I|} \int_{I} v^{1 /(p+1)} d x \equiv 1 / t
$$

If we substitute this into inequality (2) we get

$$
u(I) \leq C \frac{1}{|I|^{q}}\left(\int_{I} v^{1 /(p+1)} d x\right)^{(p+1) q / p} .
$$

Therefore

$$
\frac{u(I)}{|I|} \leq C|I|^{\gamma}\left(\frac{1}{|I|} \int_{I} v^{1 /(p+1)} d x\right)^{(p+1) q / p},
$$

where $\gamma=q / p-1>0$. Hence the right-hand side tends to 0 as $|I|$ tends to 0 . Therefore, by the Lebesgue differentiation theorem, $u(x)=0$ for almost every $x \in I_{0}$.

Now assume that for some function $f$ the right-hand side of inequality (2) is finite. We will show that the left-hand side is zero. Let $E=\{x: u(x)>0\}$. Then the left-hand side of (2) becomes $L=u(\{x \in E: m f(x)<1 / t\})$. If $I$ is an interval such that $|I \cap E|>0$, then by the previous argument, $\int_{I} v^{1 /(p+1)} d x=\infty$. Therefore, for every $x \in E^{\prime}$, the set of points in $E$ of density one, and for every $I$ such that $x \in I, \int_{I} v^{1 /(p+1)} d x=\infty$. But then for such $I$, by Hölder's inequality

$$
\begin{gathered}
\infty=\int_{I} v^{1 /(p+1)} d x=\int_{I} \frac{v^{1 /(p+1)}}{f^{p /(p+1)}} \cdot f^{p /(p+1)} d x \\
\leq\left(\int_{I} \frac{v}{f^{p}} d x\right)^{1 /(p+1)}\left(\int_{I} f d x\right)^{p /(p+1)} .
\end{gathered}
$$

Thus $\int_{I} f d x=\infty$, so $m f(x)=\infty$ for all $x \in E^{\prime}$. Hence $L \leq u\left(E / E^{\prime}\right)=$ 0 . 


\section{The Equivalence of the $\left(W_{p}\right)$ and $\left(W_{p}\right)^{*}$ Conditions}

In this section we complete the proof of Theorem 1.1 by showing that the two classes $\left(W_{p}\right)$ and $\left(W_{p}\right)^{*}$ are the same. We need the following result due to Muckenhoupt [15]. For the convenience of the reader we include the proof.

Lemma 4.1. Given a non-negative, locally integrable function $\sigma$ and an interval $I$, let $\left\{I_{\alpha}\right\}$ be a collection of intervals contained in $I$ such that, for each $\alpha, \int_{I_{\alpha}} \sigma d x \leq N\left|I_{\alpha}\right|$. If $J=\bigcup_{\alpha} I_{\alpha}$, then $\int_{J} \sigma d x \leq 2 N|J|$.

Proof: Fix $\epsilon>0$ and let $\delta>0$ be such that $\int_{E} \sigma d x<\epsilon$ whenever $|E|<\delta$ and $E \subset J$. Then there exists a finite subcollection $I_{1}, \ldots, I_{n}$, of the $I_{\alpha}$ 's such that

$$
\left|J_{n}\right| \equiv\left|\bigcup_{k=1}^{n} I_{k}\right|>|J|-\delta
$$

and no point is contained in more than two of the $I_{k}$ 's. Then

$$
\begin{aligned}
\int_{J} \sigma d x=\int_{J \backslash J_{n}} \sigma d x+\int_{J_{n}} \sigma d x \leq \epsilon & \sum_{k=1}^{n} \int_{I_{k}} \sigma d x \\
& \leq \epsilon+N \sum_{k=1}^{n}\left|I_{k}\right| \leq \epsilon+2 N|J| .
\end{aligned}
$$

Since $\epsilon$ is arbitrary we are done.

We first show that the $\left(W_{p}\right)^{*}$ condition immediately implies the $\left(W_{p}\right)$ condition. Fix an interval $I$. If $\sigma(I)=+\infty$ then the $\left(W_{p}\right)$ condition holds trivially, so assume it is finite. Then for every $x \in I, m\left(v^{1 /(p+1)} / \chi_{I}\right)(x) \leq v^{1 /(p+1)}(I)$. If $\sigma(I)=0$ then the lefthand side of the $\left(W_{p}\right)^{*}$ condition is finite only if $u(I)=0$ so again the $\left(W_{p}\right)$ condition holds trivially. Finally, if $v^{1 /(p+1)}(I)>0$ then we can substitute this into the $\left(W_{p}\right)^{*}$ condition and the $\left(W_{p}\right)$ condition follows immediately.

To prove that if $(u, v)$ is in $\left(W_{p}\right)$ then it is in $\left(W_{p}\right)^{*}$, fix $p>0$ and fix an interval $I$. We may assume that $u(I)>0$ and $\sigma(I)<\infty$ since otherwise the $\left(W_{p}\right)^{*}$ condition holds trivially. Now for any $t>0$ let $E_{t}=\left\{x \in I: m\left(\sigma / \chi_{I}\right)(x)<1 / t\right\}$. Then

$$
\int_{I} \frac{u}{m\left(\sigma / \chi_{I}\right)^{p}} d x=p \int_{0}^{\infty} t^{p-1} u\left(E_{t}\right) d t=p \int_{0}^{R}+p \int_{R}^{\infty}
$$


where $R$ will be chosen below. Since $E_{t} \subset I$, the first integral is bounded by $R^{p} u(I)$. To estimate the second, note that since $E_{t}$ is open, $E_{t}=\cup I_{k}^{t}$, where the $I_{k}^{t}$ 's are disjoint open intervals. If $x \in I_{k}^{t}$, then there exists an open interval $I_{x} \subset I_{k}^{t}$ such that

$$
\frac{1}{\left|I_{x}\right|} \int_{I_{x}} \sigma d x<1 / t
$$

Hence by Lemma 4.1,

$$
\frac{1}{\left|I_{k}^{t}\right|} \int_{I_{k}^{t}} \sigma d x<2 / t
$$

If we combine this with the $\left(W_{p}\right)$ condition we see that the second integral is dominated by

$$
\begin{aligned}
p \int_{R}^{\infty} t^{p-1} \sum_{k} u\left(I_{k}^{t}\right) d t & \leq C \int_{R}^{\infty} t^{p-1} \sum_{k}\left|I_{k}^{t}\right|^{-p} \sigma\left(I_{k}^{t}\right)^{p+1} d t \\
& \leq C \int_{R}^{\infty} t^{p-1} \sum_{k}\left|I_{k}^{t}\right| t^{-p-1} d t \\
& =C \int_{R}^{\infty} t^{-2}\left|E_{t}\right| d t
\end{aligned}
$$

Again $\left|E_{t}\right| \leq|I|$, so the last integral is bounded by $|I| / R$. Therefore

$$
\int_{I} \frac{u}{m\left(\sigma / \chi_{I}\right)^{p}} d x \leq R^{p} u(I)+C|I| / R .
$$

Let $R^{p}=\sigma(I) / u(I)$; then the first term on the right-hand side becomes $\sigma(I)$. Further, by the $\left(W_{p}\right)$ condition, $|I| / R \leq C \sigma(I)$. Since all the constants are independent of $I$ it follows that $(u, v)$ is in $\left(W_{p}\right)^{*}$ and we are done.

Remarks. In higher dimensions, the same argument shows that the $\left(W_{p}\right)^{*}$ condition implies the $\left(W_{p}\right)$ condition. The converse is true if we assume that $\sigma$ is a doubling weight. For in that case (arguing as in the remark at the end of Section 2) we have that $E_{t} \subset\left\{2 I_{x}: x \in E_{t}\right\}$, so there exists a collection of cubes $I_{k}^{t}$ with finite overlap such that $E_{t} \subset \cup 2 I_{k}^{t}$. The rest of the proof continues as before.

Recall, however, that in higher dimensions we are forced to replace the $\left(W_{p}\right)^{*}$ condition with a stronger one. (See the remarks at the end of Section 3.) To extend this part of Theorem 1.1 to higher dimensions we have to replace the $\left(W_{p}\right)$ condition by the stronger condition where the 
averages are taken over sets which are the finite union of cubes. Minor modifications to the proof in Section 2 shows that this condition is also necessary, but it is not clear if it is equivalent to the $\left(W_{p}\right)$ condition.

These modifications give a version of the equivalence of the $\left(W_{p}\right)$ and $\left(W_{p}\right)^{*}$ conditions in higher dimensions with the assumption that $\sigma$ is a doubling weight. As we noted earlier, it is not clear how this relates to the assumption (used to extend the weak-type inequality to higher dimensions) that $u$ is a doubling weight.

\section{The Structure of $\left(W_{p}\right)$ Weights}

In this section we gather together a number of observations about the structure of $\left(W_{p}\right)$ weights and their relationship to $\left(A_{p}\right)$ weights. First, we have the following inclusion.

Theorem 5.1. If $(u, v) \in\left(A_{p}\right), 1<p<\infty$, then $(u, v) \in\left(W_{q}\right)$ for all $q>0$, with constant independent of $q$. Conversely, if $u=v$ then for every $q>0,\left(W_{q}\right)=\left(A_{\infty}\right)$.

Proof: By Hölder's inequality, for all $r>1,(m f)^{-1} \leq M\left(f^{1-r^{\prime}}\right)^{r-1}$. Fix $r$ such that $q(r-1)=p_{0}>p$. Since $(u, v) \in\left(\overline{A_{p}}\right)$ implies that $(u, v) \in\left(S_{p_{0}}\right)$

$$
\begin{aligned}
\int \frac{u}{(m f)^{q}} d x \leq \int M\left(f^{1-r^{\prime}}\right)^{(r-1) q} u d x & \\
& \leq C \int\left(|f|^{1-r^{\prime}}\right)^{(r-1) q} v d x \leq C \int \frac{v}{|f|^{q}} d x .
\end{aligned}
$$

The constant $C$ depends on the $\left(A_{p}\right)$ constant of $(u, v)$ and is independent of $q$.

The proof of the converse is found in Theorem 3.1 of [2]. For completeness we include the proof here. If $(v, v) \in\left(W_{p}\right)$ then $v^{1 /(p+1)}$ satisfies a reverse Hölder inequality with exponent $p+1$. But then by a result of Strömberg and Wheeden $[\mathbf{2 5}], v=\left(v^{1 /(p+1)}\right)^{p+1} \in\left(A_{\infty}\right)$.

In the two-weight case the converse of Theorem 5.1 is false. A simple counter-example on the real line is given by the pair $\left(e^{|x|}, e^{2|x|}\right)$. In fact, between the $\left(A_{p}\right)$ and $\left(W_{q}\right)$ classes we can interpolate another class of weights. We say that the pair of weights $(u, v)$ is in $\left(W_{\infty}\right)$ if it satisfies the two-weight, reverse Jensen inequality: there exists a constant $C$ such that for every interval $I$,

$$
\frac{1}{|I|} \int_{I} u d x \leq C \exp \left(\frac{1}{|I|} \int_{I} \log v d x\right) .
$$


(If $u=v$ this inequality characterizes $\left(A_{\infty}\right)$. See García-Cuerva and Rubio de Francia [6].) This is the formal limit of the $\left(A_{p}\right)$ condition as $p \rightarrow \infty$ and the formal limit of the $\left(W_{q}\right)$ condition as $q \rightarrow \infty$. Further, it follows at once from Jensen's inequality that $\left(A_{p}\right) \subset\left(W_{\infty}\right) \subset\left(W_{q}\right)$ for all $p>1$ and all $q>0$. Both of these inclusions are proper: the pair $\left(e^{|x|}, e^{2|x|}\right)$ is in $\left(W_{\infty}\right)$ but not in any $\left(A_{p}\right)$ class; the pair $\left(e^{|x|}, e^{3|x| / 2}\right)$ is in $\left(W_{q}\right)$ for all $q>0$ but is not in $\left(W_{\infty}\right)$.

By Hölder's inequality the $\left(W_{p}\right)$ classes are themselves nested: $\left(W_{p}\right) \subset$ $\left(W_{q}\right)$ for all $p>q>0$. This inclusion is proper. To show this we will actually show more: we will construct an example to show that for any $p>0$ there exists $(u, v) \in\left(W_{p}\right)$ such that $(u, v) \notin\left(W_{p+\epsilon}\right)$ for any $\epsilon>0$. It will suffice to construct an example on $[0, \infty)$ since we can extend it to $\mathbb{R}$ as an even function.

Let $v$ be an increasing function on $[0, \infty)$ such that for any $r>0, v^{r}$ is not a doubling weight. (For example, let $v(x)=e^{-1 / x}$.) Define $u$ by the integral equation

$$
\frac{1}{t} \int_{0}^{t} u d x=\left(\frac{1}{t} \int_{0}^{t} v^{1 /(p+1)} d x\right)^{p+1} .
$$

We claim that $(u, v) \in\left(W_{p}\right)$. (Intuitively, $u$ is the largest function such that $(u, v)$ is in $\left(W_{p}\right)$.) To see this, fix $I=[a, b] \subset[0, \infty)$. Then for all $t>0$,

$$
\begin{aligned}
& m\left(v^{1 /(p+1)} / \chi_{I}\right)(t) \geq m\left(v^{1 /(p+1)}\right)(t) \\
& \quad=\frac{1}{t} \int_{0}^{t} v^{1 /(p+1)} d x=(U(t) / t)^{1 /(p+1),}
\end{aligned}
$$

where $U(t)=\int_{0}^{t} u d x$. Hence, integrating by parts we see that

$$
\begin{aligned}
\int_{I} \frac{u}{m\left(v^{1 /(p+1)} / \chi_{I}\right)^{p}} d x & \leq \int_{I} \frac{x^{p /(p+1)} u}{U^{p /(p+1)}} d x \\
& =\left.(p+1) x^{p /(p+1)} U(x)^{1 /(p+1)}\right|_{a} ^{b}-p \int_{I} \frac{U^{1 /(p+1)}}{x^{1 /(p+1)}} d x \\
& \leq\left.(p+1) \int_{0}^{x} v^{1 /(p+1)} d x\right|_{a} ^{b} \\
& =(p+1) \int_{I} v^{1 /(p+1)} d x .
\end{aligned}
$$

Therefore $(u, v)$ is in $\left(W_{p}\right)^{*}$ and so in $\left(W_{p}\right)$. 
However, $(u, v)$ cannot be in $\left(W_{p+\epsilon}\right)$ for any $\epsilon>0$. For if it were, then for all $t>0$ we would have the inequality

$$
\left(\frac{1}{t} \int_{0}^{t} v^{1 /(p+1)} d x\right)^{p+1} \leq C\left(\frac{1}{t} \int_{0}^{t} v^{1 /(p+\epsilon+1)} d x\right)^{p+\epsilon+1}
$$

Since $v$ is increasing, by Lemma 7.1 of Cruz-Uribe [1] , this reverse Hölder type inequality implies that $v^{1 /(p+\epsilon+1)}$ is in $\left(A_{\infty}\right)$ and so a doubling weight. However this contradicts our choice of $v$, so $(u, v)$ cannot be in $\left(W_{p+\epsilon}\right)$.

The property that $\left(W_{p}\right)$ implies $\left(W_{p+\epsilon}\right)$ is the analogue of the central property of $\left(A_{p}\right)$ weights when $u=v$; initially we conjectured that it held for all $\left(W_{p}\right)$ weights as well. This led to the following question.

Question 5.2. For which pairs $(u, v) \in\left(W_{p}\right)$ does there exist an $\epsilon>0$ such that $(u, v) \in\left(W_{p+\epsilon}\right)$ ?

The analogous result is known for the maximal operator: see Leckband and Neugebauer [13], [14]. Based on their work we conjecture that this problem is related to weighted norm inequalities for the iterated minimal operator.

We can summarize the above results in the chain of proper inclusions

$$
\left(A_{1}\right) \subset\left(A_{r}\right) \subset\left(A_{s}\right) \subset\left(W_{\infty}\right) \subset\left(W_{p}\right) \subset\left(W_{q}\right),
$$

$1<r<s, 0<q<p$. As we noted in the Introduction, we have the formal relationship that $\left(W_{p}\right)=\left(A_{-p}\right)$, (i.e. the $\left(A_{p}\right)$ condition with $p$ replaced by $-p)$ for all $p>0$. This lets us extend the chain of $\left(A_{p}\right)$ inclusions to negative indices.

(There is no similar relationship between the $\left(S_{p}\right)$ and $\left(W_{p}\right)^{*}$ conditions. A straightforward calculation shows that the conditions $\left(S_{-p}\right)$ and $\left(W_{-p}\right)^{*}, p>0$, are both equivalent to the class $\left.\{(u, v): u \leq C v\}.\right)$

Finally, we note that while it is easy to show that $(u, M u) \in\left(A_{1}\right)$ for any $u$, and so $(u, M u) \in\left(S_{p}\right)$ for any $p>1$, the analogous result is not true for the $\left(W_{p}\right)$ classes. Let $v(x)=e^{|x|}$. Then an easy calculation shows that $(m v, v)$ is not in $\left(W_{p}\right)$ for any $p>0$. While there ought to be some way of constructing $\left(W_{p}\right)$ weights using the minimal operator, it is unclear how to go about it.

\section{The Minimal Operator and Differentiability of the Integral}

In this section we apply the minimal operator to problems of differentiation of the integral in $\mathbb{R}^{n}$. For completeness we repeat the definitions 
given in the Introduction. Let $\mathcal{B}=\left\{\mathcal{B}_{x}: x \in \mathbb{R}^{n}\right\}$ be a differentiation basis: for each $x, \mathcal{B}_{x}=\left\{E_{j x}\right\}$ where the sets $E_{j x} \rightarrow x$-that is, for every $\epsilon>0, E_{j x} \subset\{t:|t-x| \leq \epsilon\}$ for all $j$ sufficiently large. Note that this is more general than the usual definition since we do not assume that $x \in E_{j x}$ or that $E_{j x}$ is open. Define the maximal operator relative to $\mathcal{B}$ by

$$
M_{\mathcal{B}} f(x)=\sup _{\mathcal{B}_{x}} \frac{1}{\left|E_{j x}\right|} \int_{E_{j x}}|f| d y .
$$

We will assume that $\mathcal{B}$ is such that $M_{\mathcal{B}}$ is measurable; this property is easy to check for the classical differentiation bases. (See de Guzmán [7].) If $M_{\mathcal{B}}$ is weak-type $(p, p)$ for some $1 \leq p<\infty$, then it is known that $\mathcal{B}$ differentiates $L^{p}$ : given a function $f$ in $L^{p}$, the exceptional set

$$
E_{\mathcal{B}}(f)=\left\{x: \limsup _{j \rightarrow \infty}\left|\frac{1}{\left|E_{j x}\right|} \int_{E_{j x}} f d y-f(x)\right|>0\right\}
$$

has measure zero. (See de Guzmán [7], [8].)

Throughout this section we assume that $M_{\mathcal{B}}$ is weak-type $\left(p_{0}, p_{0}\right)$, for some $p_{0} \geq 1$. We are interested in the differentiability of $\int f d x$ when $f$ is not in $L^{p_{0}}$ (that is, when $M_{\mathcal{B}} f$ is too large to give any information). In particular, we want to characterize the class of functions which $\mathcal{B}$ differentiates.

To state our main result we need to define an $\left(A_{2}\right)$-type condition relative to a differentiation basis. We say that a function $w$ is in $\left(A_{2}\right)_{\mathcal{B}}$ if

$$
A_{2 \mathcal{B}}(w) \equiv \sup _{j, x} \frac{1}{\left|E_{j x}\right|} \int_{E_{j x}} w d y \cdot \frac{1}{\left|E_{j x}\right|} \int_{E_{j x}} w^{-1} d y<\infty .
$$

(By convention we assume that $0 \cdot \infty=0$.)

Theorem 6.1. Suppose that $M_{\mathcal{B}}$ is weak-type $\left(p_{0}, p_{0}\right)$ for some $p_{0} \geq 1$. Let $f$ be a non-negative function on $\mathbb{R}^{n}$ such that $1 / f \in L^{p}\left(\mathbb{R}^{n}\right)$ for some $0<p<\infty$. Suppose that $\left\{g_{k}\right\}$ is a sequence of non-negative, measurable functions such that: $1 / g_{k}$ converges to $1 / f$ in the metric of $L^{p}$; and $A_{2 \mathcal{B}}\left(\left|f-g_{k}\right|\right) \leq C<\infty$ for all $k$. If $\left|E_{\mathcal{B}}(f)\right|>\lambda$ then for all $\epsilon>0$ there exists $k=k(\lambda, \epsilon)$ such that $\left|E_{\mathcal{B}}\left(g_{k}\right)\right|>\lambda-\epsilon$.

This theorem has the following immediate corollary.

Corollary 6.2. Under the hypotheses of Theorem 6.1, if $\left|E_{\mathcal{B}}\left(g_{k}\right)\right|=0$ for all $k$ then $\left|E_{\mathcal{B}}(f)\right|=0$.

Before proving Theorem 6.1 we make the following observations. 
First, If $\left|E_{\mathcal{B}}(f)\right|=0$ then the existence of a sequence of $g_{k}$ 's which converge in the specified manner is immediate: let $g_{k}=f+1 / k$. More generally, if $\phi$ is in $\left(A_{2}\right)_{\mathcal{B}}$ then the sequence $g_{k}=f+\phi / k$ converges in this manner to $f$.

Second, Theorem 6.1 is an example of the "transmission" of differentiability. A classical result of this kind is due to Hayes and Pauc $[\mathbf{9}]$ : if $\mathcal{B}$ is a density basis, $\mathcal{B}$ differentiates $f$ and $|g| \leq f$ then $\mathcal{B}$ differentiates $g$. To see the relation between their result and Theorem 6.1 , let $g_{k}$ be a sequence which decreases monotonically to $f$ and such that $A_{2 \mathcal{B}}\left(\left|f-g_{k}\right|\right)$ is uniformly bounded. By the monotone convergence theorem the sequence $\left\{1 / g_{k}\right\}$ converges to $1 / f$ in $L^{p}$, so by Theorem $6.1 \mathcal{B}$ differentiates $f$ if it differentiates the $g_{k}$ 's. On the other hand, if $\mathcal{B}$ is a density basis, then by the theorem of Hayes and Pauc, we have the same conclusion without having to assume the $\left(A_{2}\right)_{\mathcal{B}}$ condition. Thus Theorem 6.1 can be thought of as a generalization of their result, but the exact role of the $\left(A_{2}\right)_{\mathcal{B}}$ condition is not well understood.

Third, if $f$ is a continuous function then $E_{\mathcal{B}}(f)$ is empty. Thus, via Moore-Smith convergence techniques (see Kelley [12]) we can use the type of convergence defined in Theorem 6.1 to construct a topological space in which all functions in the closure of the continuous functions are differentiated by $\mathcal{B}$. It is reasonable to conjecture that this set contains all functions which $\mathcal{B}$ differentiates. However, we have not been able to prove this. Moreover, this topological space is not a topological vector space and it does not seem to correspond to any well-known space. Again the role of the $\left(A_{2}\right)_{\mathcal{B}}$ condition is not well understood.

To prove Theorem 6.1 we need a definition and two preliminary lemmas. Given a function $f$ define the minimal function of $f$ with respect to $\mathcal{B}$ by

$$
m_{\mathcal{B}} f(x)=\inf _{\mathcal{B}_{x}} \frac{1}{\left|E_{j x}\right|} \int_{E_{j x}}|f| d y .
$$

Lemma 6.3. There exists a constant $C$ such that for all $0<p<\infty$,

$$
\int_{\mathbb{R}^{n}} \frac{d x}{m_{\mathcal{B}}(f)^{p}} \leq C \int_{\mathbb{R}^{n}} \frac{d x}{|f|^{p}} .
$$

Proof: Since we are assuming that $M_{\mathcal{B}}$ is weak-type $\left(p_{0}, p_{0}\right)$, we know that it is strong-type $(q, q)$ for $q>p_{0}$. Using this the proof is identical to that of Theorem 5.1.

Lemma 6.4. Let $f, g$ be functions such that $f \geq 1, g \geq 1$ and 
$A_{2 \mathcal{B}}(|f-g|) \leq C_{0}<\infty$. Then

(3) $\left|\left(\frac{1}{\left|E_{j x}\right|} \int_{E_{j x}} f d y\right)^{-1}-\left(\frac{1}{\left|E_{j x}\right|} \int_{E_{j x}} g d y\right)^{-1}\right|$

$$
\leq C_{0}\left(\frac{1}{\left|E_{j x}\right|} \int_{E_{j x}}\left(\frac{f g}{|f-g|}\right)^{1 / 3} d y\right)^{-3}
$$

where if $g=+\infty$ we define $f g /|f-g|=f$.

Proof: We first consider the case when

$$
0<\int_{E_{j x}} f d x<+\infty \text { and } 0<\int_{E_{j x}} g d x<+\infty .
$$

In this case it will suffice to show that

(4)

$$
\left(\frac{1}{\left|E_{j x}\right|} \int_{E_{j x}}\left(\frac{f g}{|f-g|}\right)^{1 / 3} d y\right)^{3}\left|\frac{1}{\left|E_{j x}\right|} \int_{E_{j x}} f d y-\frac{1}{\left|E_{j x}\right|} \int_{E_{j x}} g d y\right|
$$

is less than or equal to

$$
C_{0} \frac{1}{\left|E_{j x}\right|} \int_{E_{j x}} f d y \cdot \frac{1}{\left|E_{j x}\right|} \int_{E_{j x}} g d y .
$$

If we apply Hölder's inequality twice to (4) we see that it is dominated by

$$
\begin{aligned}
\left(\frac{1}{\left|E_{j x}\right|} \int_{E_{j x}} f d y\right) & \left(\frac{1}{\left|E_{j x}\right|} \int_{E_{j x}} g d y\right) \\
& \left(\frac{1}{\left|E_{j x}\right|} \int_{E_{j x}} \frac{d y}{|f-g|}\right)\left(\frac{1}{\left|E_{j x}\right|} \int_{E_{j x}}|f-g| d y\right),
\end{aligned}
$$

and by our hypothesis the product of the last two factors is at most $C_{0}$.

Now suppose that neither $f$ nor $g$ is integrable on $E_{j x}$. Then the left-hand side of (3) is 0 so there is nothing to prove.

Finally, suppose that

$$
\int_{E_{j x}} f d x<\infty \text { but } \int_{E_{j x}} g d x=+\infty .
$$


Then

$$
\int_{E_{j x}}|f-g| d x=+\infty
$$

so by the $\left(A_{2}\right)_{\mathcal{B}}$ condition

$$
\int_{E_{j x}} \frac{d x}{|g-f|}=0
$$

Hence $|g-f|=+\infty$ almost everywhere on $E_{j x}$ and, since we assume that $f$ is finite almost everywhere on $E_{j x}$, this implies that $g=+\infty$ almost everywhere on $E_{j x}$. But then inequality (3) reduces to Hölder's inequality.

Remark. The proof of Lemma 6.4 makes strong use of the $\left(A_{2}\right)_{\mathcal{B}}$ condition: we cannot replace it with the weaker condition $\left(A_{p}\right)_{\mathcal{B}}, p>2$.

We can now prove Theorem 6.1. Since $E_{\mathcal{B}}(f)=E_{\mathcal{B}}(f+1)$ and $1 /(f+1) \in L^{p}$ if $1 / f$ is, we may assume without loss of generality that $f \geq 1$. For $i>0$ define

$$
E_{i}=\left\{x: \limsup _{j}\left|\left(\frac{1}{\left|E_{j x}\right|} \int_{E_{j x}} f d y\right)^{-1}-\frac{1}{f(x)}\right|>1 / i\right\} .
$$

Then the $E_{i}$ 's are nested and their union is all of $E_{\mathcal{B}}(f)$. Therefore we may choose $i$ sufficiently large that $\left|E_{i}\right|>\lambda$. Now for any $k$, by Lemma 6.4

$$
\begin{aligned}
& \limsup _{j}\left|\left(\frac{1}{\left|E_{j x}\right|} \int_{E_{j x}} f d y\right)^{-1}-\frac{1}{f(x)}\right| \\
\leq & \limsup _{j}\left|\left(\frac{1}{\left|E_{j x}\right|} \int_{E_{j x}} f d y\right)^{-1}-\left(\frac{1}{\left|E_{j x}\right|} \int_{E_{j x}} g_{k} d y\right)^{-1}\right| \\
& +\limsup _{j}\left|\left(\frac{1}{\left|E_{j x}\right|} \int_{E_{j x}} g_{k} d y\right)^{-1}-\frac{1}{g_{k}(x)}\right|+\left|\frac{1}{g_{k}(x)}-\frac{1}{f(x)}\right| \\
\leq & C\left(m_{\mathcal{B}}\left(\frac{f g_{k}}{\left|f-g_{k}\right|}\right)^{1 / 3}(x)\right)^{-3} \\
& +\limsup _{j}\left|\left(\frac{1}{\left|E_{j x}\right|} \int_{E_{j x}} g_{k} d y\right)^{-1}-\frac{1}{g_{k}(x)}\right|+\left|\frac{1}{g_{k}(x)}-\frac{1}{f(x)}\right| \\
= & A_{k}(x)+B_{k}(x)+C_{k}(x) .
\end{aligned}
$$


Therefore

$$
E_{i} \subset\left\{x: A_{k}(x)>1 / 3 i\right\} \cup\left\{x: B_{k}(x)>1 / 3 i\right\} \cup\left\{x: C_{k}(x)>1 / 3 i\right\},
$$

so by Lemma 6.3 (with exponent $3 p$ ),

$$
\begin{aligned}
\left|E_{i}\right| \leq & (3 i C)^{p} \int_{\mathbb{R}^{n}} \frac{d y}{m_{\mathcal{B}}\left(\left(\frac{\left|f g_{k}\right|}{\left|f-g_{k}\right|}\right)^{1 / 3}\right)^{3 p}} \\
& +\left|E\left(g_{k}\right)\right|+(3 i)^{p} \int_{\mathbb{R}^{n}}\left|\frac{1}{g_{k}}-\frac{1}{f}\right|^{p} d y \\
\leq & (3 i C)^{p} \int_{\mathbb{R}^{n}} \frac{d y}{\left(\frac{\left|f g_{k}\right|}{\left|f-g_{k}\right|}\right)^{p}}+\left|E\left(g_{k}\right)\right|+(3 i)^{p} \int_{\mathbb{R}^{n}}\left|\frac{1}{g_{k}}-\frac{1}{f}\right|^{p} d y \\
= & (3 i)^{p}\left(C^{p}+1\right) \int_{\mathbb{R}^{n}}\left|\frac{1}{g_{k}}-\frac{1}{f}\right|^{p} d y+\left|E\left(g_{k}\right)\right| .
\end{aligned}
$$

Now choose $k$ so large that the first term is less than $\epsilon$, and the proof is complete.

\section{Weak-Type Inequalities for the Maximal Operator}

In this section we give a condition on a collection of sets $\mathcal{B}=\left\{E_{j}\right\}$ such that $E_{j} \rightarrow 0$ so that $\mathcal{B}$ has a subsequence $\left\{E_{j_{k}}\right\}$ such that the basis $\mathcal{B}^{*}=\left\{\mathcal{B}_{x}=\left\{E_{j_{k}}+x\right\}\right\}$ differentiates $L^{p}, p \geq 1$. (For brevity we will write $\mathcal{B}^{*}=\left\{E_{j_{k}}\right\}$.) In order to state our main result, we need a definition. If $E \subset \mathbb{R}^{n}$, define the set $E^{*}=E-E=\{x-y: x, y \in E\}$. The measure of $E^{*}$ could be much larger than that of $E$, though in some cases it is not. For example, if $E$ is convex, then as a consequence of the Brunn-Minkowski theorem, $\left|E^{*}\right| \leq\left(\begin{array}{c}2 n \\ n\end{array}\right)|E|$. (For details, see Schneider $\left[\mathbf{2 1}\right.$, p. 409].) We show that if the $E_{j}$ 's are such that $\left|E_{j}^{*}\right| \leq C\left|E_{j}\right|$ then a "good" subsequence can always be extracted from it. More precisely, we prove the following.

Theorem 7.1. Let $\mathcal{B}=\left\{E_{j}\right\}$ be a collection of sets such that $E_{j} \rightarrow 0$. Then there exists a subsequence $\mathcal{B}^{*}=\left\{E_{j_{k}}\right\}, j_{k}<j_{k+1}$ for all $k$, such that the maximal function

$$
M^{*} f(x)=\sup _{k} \frac{1}{\left|E_{j_{k}}^{*}\right|} \int_{E_{j_{k}}+x}|f| d y
$$


is weak $(1,1)$. In particular, if there exists a constant $C$ such that $\left|E_{j}^{*}\right| \leq C\left|E_{j}\right|$ for all $j$, then the maximal operator $M_{\mathcal{B}^{*}}$ is weak $(1,1)$. Thus it is also weak $(p, p)$ for $p \geq 1$, and so $\mathcal{B}^{*}$ differentiates $L^{p}$.

Before proving Theorem 7.1, we first make some observations. Consider the special case where $E_{j}=B\left(x_{j}, r_{j}\right)$, that is, each $E_{j}$ is a ball of radius $r_{j}$ and center $x_{j}$. If the ratio $\left|x_{j}\right| / r_{j}$ is uniformly bounded, then the balls approach the origin non-tangentially. This is the classical case, and the maximal operator $M_{\mathcal{B}}$ is dominated by the Hardy-Littlewood maximal operator and so is always weak $(1,1)$. (See Stein [24].) If the ratio $\left|x_{j}\right| / r_{j} \rightarrow \infty$ then the balls approach the origin tangentially. In this case there is always a subsequence of the balls which forms a differentiation basis. This extends results by Nagel and Stein [16], [24], who showed that there exists a differentiation basis of balls approaching the origin non-tangentially.

The existence of such a subsequence is the best possible result, as the following example shows. We will construct a sequence of balls $\left\{B_{j}\right\}$, $B_{j} \rightarrow 0$, and a function $f \in L^{\infty}$ such that

$$
\frac{1}{\left|B_{j}\right|} \int_{B_{j}} f(x+y) d y \nrightarrow f(x),
$$

for all $x$ in a set of positive measure. Let $C$ be a compact, nowhere dense subset of $\mathbb{R}^{2}$ of positive measure. We construct the balls $\left\{B_{j}\right\}$ as follows. Fix $n>0$ and let $\sigma_{1}, \ldots, \sigma_{k}$ be a finite set of balls of radius $1 / n$ such that $C \subset \sigma_{1} \cup \cdots \cup \sigma_{k}$. For $1 \leq i \leq k$, let $\sigma_{i}^{\prime}$ be the ball concentric with $\sigma_{i}$ of radius $1 /(n+1)$. Now choose a closed ball $A_{i}$ in $\left(\sigma_{i} \backslash \sigma_{i}^{\prime}\right)^{o}$ such that $A_{i} \cap C=\emptyset$. For each $x \in \bar{\sigma}_{i} \cap C$ there exists $\eta_{x}>0$ such that, if $|z-x| \leq \eta_{x}$ then $A_{i}+(z-x) \subset\left(\sigma_{i} \backslash \sigma_{i}^{\prime}\right)^{o}$ and $\left(A_{i}+(z-x)\right) \cap C=\emptyset$. For each $i$, the collection of balls $\left\{\sigma\left(x, \eta_{x}\right): x \in C \cap \bar{\sigma}_{i}\right\}$ covers $C \cap \bar{\sigma}_{i}$, so there exists a finite subcover $\left\{\sigma\left(x_{j}, \eta_{j}\right): 1 \leq j \leq k_{i}\right\}$. We form the collection of balls $\left\{A_{i}-x_{j}, 1 \leq j \leq k_{i}, 1 \leq i \leq k\right\}$. The union of all such sets of balls over all $n$ gives a sequence of balls $\left\{B_{j}\right\}$.

Now for each $x \in C$ there is a subsequence $B_{j_{i}} \rightarrow 0$ such that $\left(x+B_{j_{i}}\right) \cap C=\emptyset$. For at the $n$-th stage of our construction $x \in \sigma_{i}$ for some $1 \leq i \leq k$, so $x \in \sigma\left(x_{j}, \eta_{x_{j}}\right)$. Hence $A_{i}-x_{j}+x$ is a ball in $\sigma_{i} \backslash \sigma_{i}^{\prime}$ which is disjoint from $C$. Further, $A_{i}-x_{j} \subset\{|x| \leq 2 / n\}$. Now let $f=\chi_{C}$; then

$$
\frac{1}{\left|B_{j_{i}}\right|} \int_{B_{j_{i}}} f(x+y) d y=0 .
$$

This shows that $\left\{B_{j}\right\}$ is the desired sequence. 
Finally, we note that we have relied on the fact that balls are convex. This raises the following question, for which we have no good intuition as to the correct answer.

Question 7.2. Given a collection of sets $\mathcal{B}=\left\{E_{j}\right\}, E_{j} \rightarrow 0$, does a "good" subsequence always exist without the hypothesis that $\left|E_{j}^{*}\right| \leq C\left|E_{j}\right|$ ?

We now prove Theorem 7.1. To do so we need two lemmas.

Lemma 7.3. If $E \subset \mathbb{R}^{n}$ is compact and $\left|E^{*}\right|>0$, then there exists $\delta>0$ such that $\left|\left(B_{\delta} \cup E\right)^{*}\right| \leq 3\left|E^{*}\right|$, where $B_{\delta}=\{x:|x| \leq \delta\}$.

Proof: Let $\delta_{j}=1 / j$. Then $\left|\left(B_{\delta_{j}} \cup E\right)^{*}\right| \rightarrow\left|\cap_{j}\left(B_{\delta_{j}} \cup E\right)^{*}\right|$. Furthermore,

$$
\bigcap_{j}\left(B_{\delta_{j}} \cup E\right)^{*}=(\{0\} \cup E)^{*} .
$$

One inclusion is obvious. To see the reverse inclusion, let $z \in \cap_{j}\left(B_{\delta_{j}} \cup\right.$ $E)^{*}$. Then for each $j, z=x_{j}-y_{j}$ for some $x_{j}, y_{j} \in B_{\delta_{j}} \cup E$. There are three cases. If $x_{j}, y_{j} \in E$ for some $j$ then $z \in E^{*} \subset(\{0\} \cup E)^{*}$. If there exist an infinite number of $j$ 's such that $x_{j} \in B_{\delta_{j}}$, then (by passing to a subsequence) $x_{j} \rightarrow 0$. Since $E$ is compact (again passing to a subsequence) $y_{j} \rightarrow y$, where $y \in\{0\} \cup E$. Hence $z=0-y \in(\{0\} \cup E)^{*}$. By symmetry the same argument holds if an infinite number of $y_{j}$ 's are in $B_{\delta_{j}}$. To complete the proof, note that $\left|(\{0\} \cup E)^{*}\right| \leq\left|E^{*}\right|+2|E|<3\left|E^{*}\right|$, and so the desired inequality holds if we choose $\delta$ sufficiently small.

Lemma 7.4. Let $\left\{E_{j}\right\}$ be a sequence of sets such that $0<\left|E_{j}\right|<\infty$, and let $\epsilon_{j}=\left|E_{j}\right| / 2^{j}$. If $C_{j} \subset E_{j}$ with $\left|C_{j}\right| \leq \epsilon_{j}$, then

$$
M_{*} f(x)=\sup _{j} \frac{1}{\left|E_{j}\right|} \int_{C_{j}+x}|f| d y
$$

satisfies $\left\|M_{*} f\right\|_{p} \leq\|f\|_{p}, p \geq 1$.

Proof: Clearly

$$
M_{*} f(x) \leq \sum_{j \geq 1} \frac{1}{\left|E_{j}\right|} \int_{C_{j}}|f(x+y)| d y,
$$

so by Minkowski's integral inequality we see that

$$
\left\|M_{*} f\right\|_{p} \leq\|f\|_{p} \sum \frac{\left|C_{j}\right|}{\left|E_{j}\right|} \leq\|f\|_{p} .
$$


We can now prove Theorem 7.1. First suppose that each set $E_{j}$ is compact. We construct the desired subsequence using Lemma 7.3 as follows: Let $j_{1}=1$. Choose $\delta_{1}>0$ such that $\left|\left(B_{\delta_{1}} \cup E_{1}\right)^{*}\right| \leq 3\left|E_{1}^{*}\right|$. Now fix $j_{2}>j_{1}$ such that $E_{j} \subset B_{\delta_{1}}, j \geq j_{2}$, and choose $0<\delta_{2} \leq \delta_{1}$ such that $\left|\left(B_{\delta_{2}} \cup E_{j_{2}}\right)^{*}\right| \leq 3\left|E_{j_{2}}^{*}\right|$. If we continue this process we obtain a subsequence $\left\{E_{j_{k}}\right\}$ with the property that for all $k \geq 1$,

$$
\left|\left(\bigcup_{i \geq k} E_{j_{i}}\right)^{*}\right| \leq 3\left|E_{j_{k}}^{*}\right| \text {. }
$$

Define a monotone family of sets by $\mathcal{F}=\left\{S_{k}: S_{k}=\cup_{i>k} E_{j_{i}}\right\}$. Then the associated maximal function

$$
M_{\mathcal{F}}^{*} f(x)=\sup _{k} \frac{1}{\left|S_{k}^{*}\right|} \int_{S_{k}+x}|f| d y
$$

is weak $(1,1)$. (See Neugebauer [17].) But an easy estimate shows that $M^{*} f(x) \leq 3 M_{\mathcal{F}}^{*} f(x)$, which completes the proof of the special case.

For arbitrary sets $E_{j}$, let $K_{j} \subset E_{j}$ be compact subsets such that $\left|C_{j}\right|=\left|E_{j} \backslash K_{j}\right| \leq \epsilon_{j}$, where $C_{j}$ and $\epsilon_{j}=\left|E_{j}\right| / 2^{j}$ are as in Lemma 7.4. Then there is a subsequence $\left\{E_{j_{k}}\right\}$ such that the maximal function

$$
M^{* *} f(x)=\sup _{k} \frac{1}{\left|K_{j_{k}}^{*}\right|} \int_{K_{j_{k}}+x}|f| d y
$$

is weak $(1,1)$. The maximal function $M^{*} f(x)$ satisfies $M^{*} f(x) \leq$ $M^{* *} f(x)+M_{*} f(x)$, so by Lemma 7.4 it is also weak $(1,1)$.

\section{References}

1. D. Cruz-URibe, SFO, Piecewise monotonic doubling measures, Rocky Mountain J. Math. 26(2) (1996), 1-39.

2. D. Cruz-Uribe, SFO and C. J. Neugebauer, The structure of the reverse Hölder classes, Trans. Amer. Math. Soc. 347 (1995), 2941-2960.

3. D. Cruz-Uribe, SFO And C. J. Neugebauer, Weighted norm inequalities for the geometric maximal operator, Publ. Mat. (to appear).

4. D. Cruz-Uribe, SFO, C. J. Neugebauer and V. Olesen, The one-sided minimal operator and the one-sided reverse Hölder inequality, Studia Math. 116 (1995), 255-270. 
5. D. Cruz-Uribe, SFO, C. J. Neugebauer and V. Olesen, Weighted norm inequalities for a family of one-sided minimal operators, Illinois J. Math. 41 (1997), 77-92.

6. J. García-Cuerva And J. L. Rubio DE Francia, "Weighted Norm Inequalities and Related Topics," North Holland Math. Studies 116, North Holland, Amsterdam, 1985.

7. M. DE Guzmán, "Differentiation of integrals in $\mathbb{R}^{n}$," Lecture Notes in Mathematics 481, Springer-Verlag, New York, 1975.

8. M. DE GuZmán, "Real variable methods in Fourier analysis," North-Holland Math. Studies 46, North-Holland, Amsterdam, 1981.

9. C. Hayes and C. PAuc, Full individual and class differentiation theorems in their relation to halo and Vitali properties, Canad. J. Math. 7 (1955), 221-274.

10. T. IwANIEC AND C. Nolder, Hardy-Littlewood inequality for quasiregular mappings in certain domains of $\mathbb{R}^{n}$, Ann. Acad. Sci. Fenn. Ser. A I Math. 10 (1985), 267-282.

11. B. JAWERTH, Weighted inequalities for maximal operators: linearization, localization and factorization, Amer. J. Math. 108 (1986), 361-414.

12. J. Kelley, "General Topology," Van Nostrand, New York, 1955.

13. M. A. Leckband And C. J. Neugebauer, A general maximal operator and the $\left(A_{p}\right)$ condition, Trans. Amer. Math. Soc. 275(2) (1983), 821-831.

14. M. A. Leckband And C. J. Neugebauer, Weighted iterates and variants of the Hardy-Littlewood maximal operator, Trans. Amer. Math. Soc. 279(1) (1983), 51-61.

15. B. Muckenhoupt, Weighted norm inequalities for the Hardy maximal function, Trans. Amer. Math. Soc. 165 (1972), 207-226.

16. A. Nagel And E. M. Stein, On certain maximal functions and approach regions, Adv. Math. 54 (1984), 83-106.

17. C. J. Neugebauer, On the Hardy-Littlewood maximal function and some applications, Trans. Amer. Math. Soc. 259 (1980), 99-105.

18. E. SAWYER, A characterization of a two weight norm inequality for maximal operators, Studia Math. 75 (1982), 1-11.

19. E. SAWYER, Two weight norm inequalities for certain maximal and integral operators, in "Harmonic Analysis," Proceedings of a conference held at the University of Minnesota, Minneapolis, April 20-30, 1981 (Co. Weiss and F. Ricci, eds.), Lecture Notes in Mathematics 908, Springer Verlag, New York, 1982, pp. 102-127. 
20. C. Sbordone And I. WiK, Maximal functions and related weight classes, Publ. Mat. 38 (1994), 127-155.

21. R. Schneider, "Convex Bodies: The Brunn-Minkowski Theory," Encyclopedia of Mathematics and its Applications, Cambridge University Press, Cambridge, 1993.

22. P. SJÖGREN, A remark on the maximal function for measures on $\mathbb{R}^{n}$, Amer. J. Math. 105 (1983), 1231-1233.

23. E. M. Stein, "Singular Integrals and Differentiability Properties of Functions," Princeton University Press, Princeton, 1970.

24. E. M. Stein, "Harmonic Analysis Real Variable Methods, Orthogonality, and Oscillatory Integrals," Princeton University Press, Princeton, 1993.

25. J. O. Strömberg And R. L. Wheeden, Fractional integrals on weighted $H^{p}$ and $L^{p}$ spaces, Trans. Amer. Math. Soc. 287 (1985), 293-321.

26. A. M. Vargas, On the maximal function for rotation invariant measures in $\mathbb{R}^{n}$, Studia Math. 110 (1994), 9-17.

27. X. Yin And B. Muckenhoupt, Weighted inequalities for the maximal geometric mean operator, Proc. Amer. Math. Soc. 124 (1996), 75-81.

David Cruz-Uribe:

Department of Mathematics

Trinity College

Hartford, CT 06106-3100

U.S.A.

e-mail: david.cruzuribe@mail.trincoll.edu

\author{
C. J. Neugebauer: \\ Department of Mathematics \\ Purdue University \\ West Lafayette,IN 47907-1395 \\ U.S.A. \\ e-mail: neug@math.purdue.edu
}

V. Olesen:
Department of Mathematics
Purdue University
West Lafayette,IN 47907-1395
U.S.A.
e-mail: olesen@math.purdue.edu

Primera versió rebuda el 2 de Setembre de 1996, darrera versió rebuda el 22 de Juliol de 1997 